\title{
Une nouvelle méthode pour la mesure du flux de sève brute dans le tronc des arbres
}

\author{
A. GRANIER \\ I.N.R.A.C.R.F., Station de Sylviculture et de Production \\ Champenoux, F 54280 Seichamps
}

\begin{abstract}
Résumé
La méthode décrite dans cet article utilise un capteur thermique composé de deux sondes insérécs radialement dans le bois d'aubier du tronc. Une de ces sondes est chauffée à puissance constante, l'autre sert de référence de température. Une équation simple permet de calculer le flux de sève en fonction de la différence de température entre les deux éléments. Un étalonnage a été effectué sur des échantillons de tronc de différentes essences.
\end{abstract}

Le faible coût et la sensibilité de ce système doivent permettre d'aborder la mesure quantitative de la transpiration et de sa variabilité en forêt.

La mesure de la transpiration constitue un élément essentiel de la compréhension de la physiologie de l'arbre et de la dynamique des transferts d'eau dans les peuplements forestiers. Dans le cadre des recherches menées à la Station de Sylviculture et de Production du C.R.F., nous nous sommes intéressés depuis plusieurs années à la mesure du flux de sève brute dans le tronc des arbres. Il existe à ce jour de nombreuses méthodes de mesure du flux transpiratoire; la méthode des impulsions de chaleur a eu, depuis Hüber \& Schmidt (1937) un développement très significatif, notamment grâce à SWANSON $(1962,1974)$. Nous avons largement utilisé cette technique, en la rendant automatique, et en modifiant la structure du capteur (GraniER $\&$ Gross, en cours de publication).

Toutefois, aucune de ces méthodes ne présente les spécifications requises pour permettre, à faible coût, de procéder à un échantillonnage de la transpiration des arbres en peuplements forestiers. La méthode des impulsions de chaleur a en particulier deux inconvénients : son caractère ponctuel dans le tronc et son imprécision: en condition de transpiration faible.

Nous proposons ici une nouvelle méthode et un capteur permettant de mesurer le flux de sève suivant un axe radial dans le bois d'aubier du tronc. 

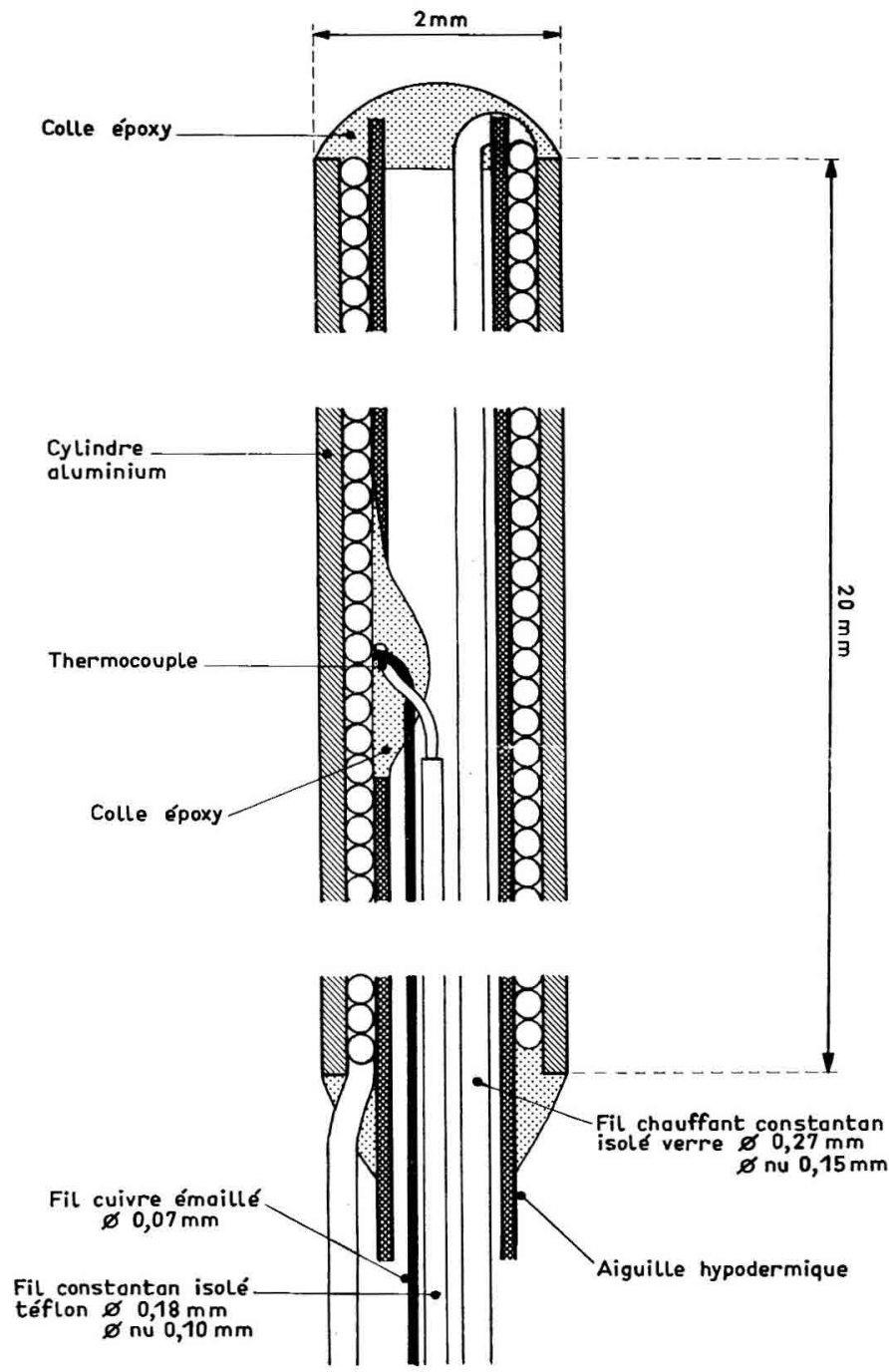

ECHELLE $12,5: 1$

FIG. 1

Coupe longitudinale de l'élément chauffant.

Longitudinal sectional view of the heating probe. 


\section{Matériel et méthodes}

\subsection{Matériel}

Le capteur (fig. 1) se compose de deux sondes cylindriques de $2 \mathrm{~mm}$ de diamètre et de $20 \mathrm{~mm}$ de longueur, insérées radialement dans le bois d'aubier du tronc, séparées d'une distance de $5 \mathrm{~cm}$ environ. Une de ces sondes est chauffée à puissance constante au moyen d'un enroulement de fil de constantan autour d'une aiguille hypodermique en acier inoxydable. La résistance chauffante est calibrée à $10 \Omega$ et la tension d’alimentation est délivrée par une alimentation stabilisée réglable. Un thermocouple (cuivre - constantan) est disposé à mi-longueur de la résistance chauffante et une gaine en aluninium recouvre l'ensemble pour en uniformiser la température. La deuxième sonde, située dans le tronc au-dessous de la précédente, renferme un thermocouple identique et monté en opposition avec celui de l'élément chauffant. Le système permet ainsi de mesurer l'écart de température entre les deux sondes.

Un amplificateur opérationnel de type OP 27 PMI permet d'augmenter le signal des thermocouples (avec un gain de 1000 ).

\subsection{Théorie}

En condition de régime thermique établi entre lélément chauffant et le milieu (bois + sève), et pour un flux de sève constant, nous supposerons que lapport de chaleur par effet Joule est égal à la quantité de chaleur dissipée au niveau de la paroi du capteur. Nous posons donc :

$$
\mathrm{hS}\left(\mathrm{T}-\mathrm{T}_{\infty}\right)=\mathrm{R} \mathrm{i}^{2}
$$

avec :

$\mathrm{h}=$ coefficient de transfert de la chaleur $\left(\mathrm{W}, \mathrm{m}^{-2} . " \mathrm{C} 1\right)$,

$S=$ aire de la surface d'échange $\left(\mathrm{m}^{2}\right)$,

$\mathrm{T}=$ température du cylindre $\left({ }^{\circ} \mathrm{C}\right)$,

$\mathrm{T}_{\infty}=$ température du matériau bois en l'absence de chauffage ("C),

$\mathrm{R}=$ résistance électrique $(\Omega)$,

$\mathrm{i}=$ intensité du courant électrique (A). forme :

Le coefficient $\mathrm{h}$ est supposé dépendre du débit de la sève $\mathrm{u}(\mathrm{m} . \mathrm{s})$ sous la

$$
h=h_{o}(1+\alpha \cdot u)
$$

où $h_{0}$ est le coefficient d'échange lorsque $u=0$ (transpiration nulle), qu'il est possible de calculer d'après (1) :

$$
h_{o}=\frac{\mathrm{Ri}^{2}}{S\left(\mathrm{~T}_{\mathrm{M}}-\mathrm{T}_{\omega}\right)}
$$

$\mathrm{T}_{\mathrm{M}}$ désigne la température à flux de sève nul $(\mathrm{u}=0)$ 
Lorsque $\mathrm{u}$ est constant et non nul, il vient :

$$
\mathrm{u}=\frac{1}{i} \frac{\mathrm{T}_{\mathrm{M}}-\mathrm{T}}{\mathrm{T}-\mathrm{T}_{\infty}}
$$

Le rapport $\frac{T_{M}-T}{T-T_{\infty}}$ est un nombre sans dimension, que nous appellerons index de flux $K$, proportionnel à $u$.

\section{Résultats}

\subsection{Etalonnage}

Le capteur a été testé et étalonné sur des fragments de troncs de diamètre compris entre 40 et $50 \mathrm{~mm}$. Nous avons fait circuler de l'eau sous pression dans ces échantillons, et mesuré simultanément le débit de l'eau par pesée de l'exsudat et le signal

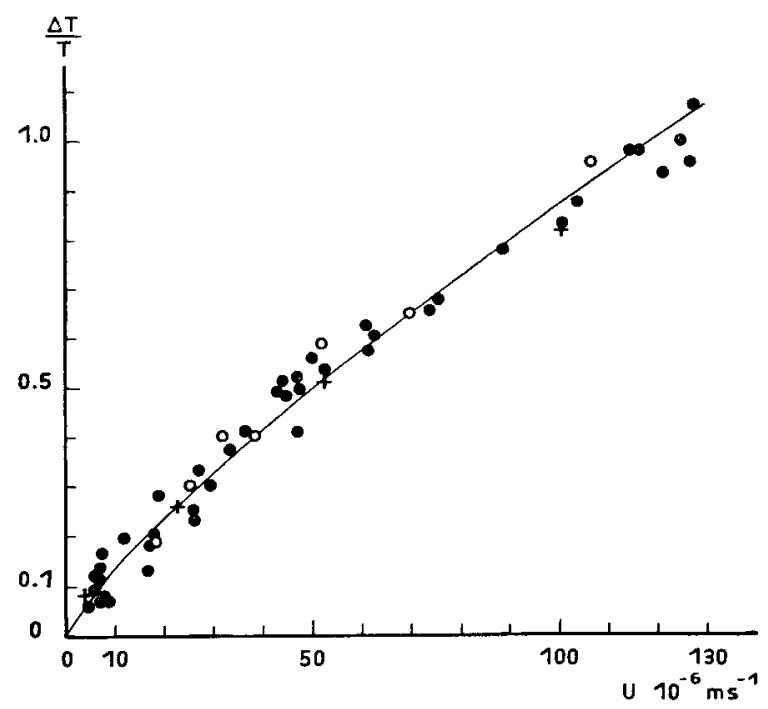

- Pseudotsuga menziesii

- Pines nigra

+ Quercus pedunculata

FIG. 2

Relation entre le rapport $K$ (voir texte $\$ 1.2$ ) et le flux d'eau par unité de section de bois d'aubier pour différentes essences.

Relationship between $K$ (see text $\$ 1.2$ ) and the water flux per unit of sapwood area, for different species. 
délivré par le capteur thermique. Le débit pouvait être modifié en ajustant la pression de l'eau. Après les mesures, chaque échantillon était sectionné pour mesurer la section du bois d'aubier au niveau de l'élément chauffant. L'étalonnage a porté sur trois essences différentes : douglas, pin noir, et chêne pédonculé.

La figure 2 représente les résultats obtenus, avec en abscisse le flux d'eau par unité de surface $\mathbf{u}\left(\mathrm{en} \mathrm{m} . \mathrm{s}^{-1}\right.$ ) et en ordonnée le rapport $\mathrm{K}$. Le calcul de $\mathrm{K}$ s'effectue d'après la relation (4) en connaissant pour chaque échantillon la température $T_{M}$ atteinte lorsque le flux d'eau est nul. Il est intéressant de constater que la relation entre $\mathbf{K}$ et $\mathbf{u}$ est la même pour les trois espèces; le coefficient $\alpha$ de l'équation (4) semble donc, dans les conditions expérimentales, indépendant de l'essence. Un ajustement non linéaire a conduit à la relation expérimentale :

$$
\mathrm{K}=0.0206 \mathbf{u}^{0.8124}
$$

où u est exprimé en $10^{-6} \mathrm{~m} \cdot \mathrm{s}^{-1}$.

$$
\begin{aligned}
& \mathrm{r}^{2}=0.96 \\
& \mathrm{n}=53 \text { points }
\end{aligned}
$$

Signalons enfin que nous avons fixé l'intensité du courant dans la résistance chauffante à une valeur de $0,141 \mathrm{~A}$, ce qui est un compromis entre la sensibilité du capteur (qui augmente avec l'intensité appliquée) et le risque d'un échauffement de la sonde de référence de la température (1).

\subsection{Mesures sur des arbres}

Durant l'été et l'automne 1983, nous avons effectué des enregistrements journaliers de flux de transpiration sur des douglas de la forêt domaniale d'Amance (à $15 \mathrm{~km}$ à l'est de Nancy). Nous avons choisi des arbres placés dans des situations différentes : des arbres de plein découvert, d'une hauteur de $5 \mathrm{~m}$, et des arbres d'une plantation régulière âgée de 20 ans, pour une hauteur moyenne de $15 \mathrm{~m}$. Quatre arbres (deux dans chacune des situations) ont été équipés chacun d'un capteur thermique inséré radialement dans leur bois d'aubier.

Le coefficient $\mathrm{K}$ repose sur l'évaluation de $T_{\mathrm{XI}}$ (voir équation (4) au $\$ 1.2$ ). Nous avons supposé que cette température d'équilibre à flux de sève nul pouvait être mesurée lors de séquences nocturnes où l'humidité de l'air est voisine de la saturation.

La figure 3 reporte pour une journée (le 16-8-1983) la variation du coefficient $K$, observée pour un arbre de chaque traitement, ainsi que l'évolution de l'évapotranspiration potentielle (E.T.P.) calculée par la formule de Penman. Nous avons choisi une journće présentant des passages nuageux qui permettent de mettre en évidence la réponse des capteurs. Les deux arbres suivent une évolution similaire, en parículier au niveau des maximas et des minimas. Le retard important de $\mathrm{K}$ par rapport à l'E.T.P. le matin correspond à une phase d'évaporation de la rosée sur les aiguilles. Après cette phase, le facteur $K$ des deux arbres suit la variation de l'E.T.P. Il est à noter que la chute brutale de l'E.T.P. intervenant en milieu de journée (passages nuageux) se manifeste de façon plus accentuée pour l'arbre situé en plein découvert. L'arbre de peuplement semble ainsi mieux tamponner les variations de l'E.T.P. En fin de journée, si les deux arbres voient diminuer $K$ en même temps que I'E.T.P., un retard se manifeste pour le douglas du peuplement.

(1) D'après nos mesures, le coefficient $\alpha$ des relations (2) et (4) est dépendant de la quantité de chaleur apportée. 


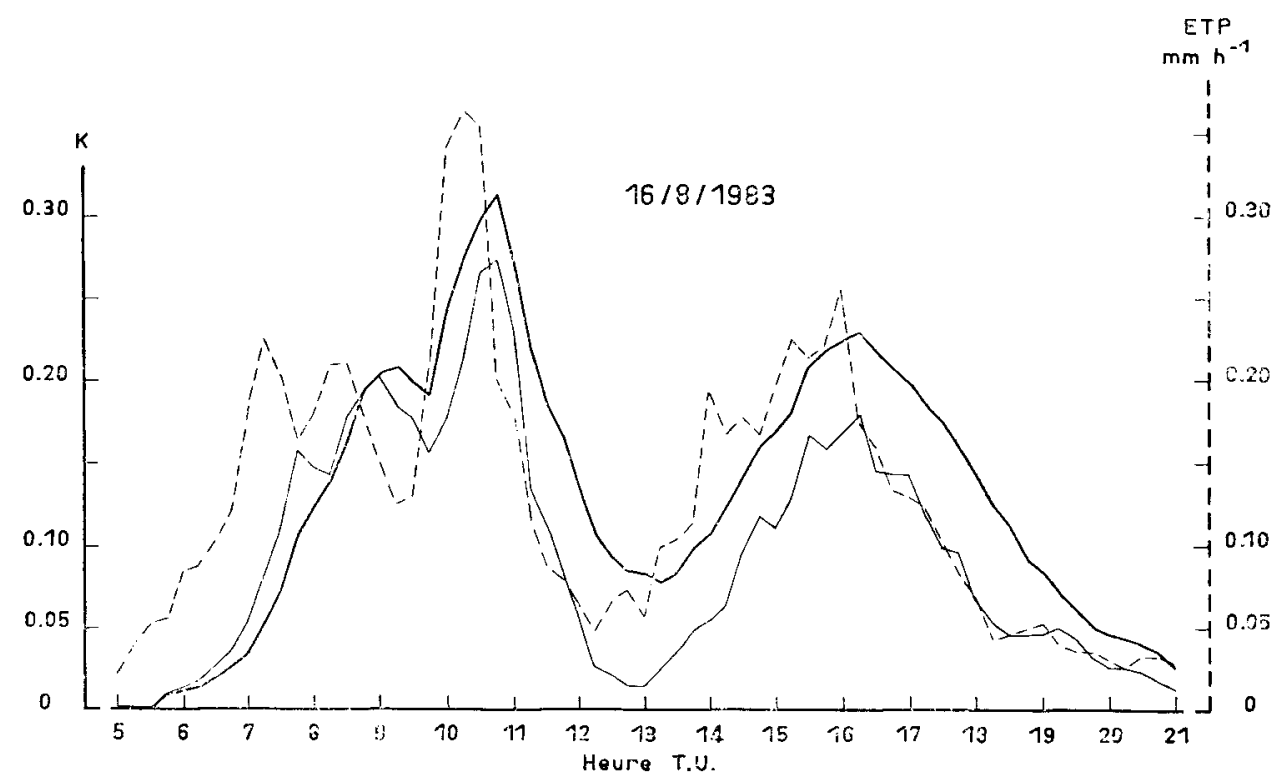

FIG. 3

Evolution du rapport $K$ pour deux Douglas et de l'E.T.P. Penman au cours d'une journée. Diurnal evolution of $K$ for two Douglas fir trees and of Penman P.E.T.

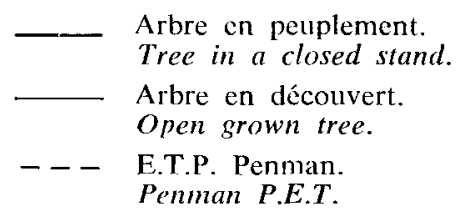

Un nombre important de journées a ainsi été étudié : nous avons représenté sur la figure 4, pour l'ensemble des journées, la valeur du coefficient $\mathrm{K}_{2.1}$ de l'arbre en peuplement, en fonction de l'E.T.P. journalière exprimée en $\mathrm{mm}$. Le coefficient $\mathrm{K}_{24}$ est égal à la moyenne journalière de $\mathrm{K}$ défini d'après l'équation (4).

Pendant la durée de ces mesures, l'arbre étudié a été en permanence bien alimenté en eau; la transpiration et donc le coefficient $K_{w_{4}}$ sont donc sensiblement proportionnels à l'E.T.P. Nous avons aussi fait figurer les journées présentant des épisodes pluvieux diurnes, quelles que soient leur durée et leur intensité. Certaines de ces journćes s'écartent du nuage de points : l'interception de l'eau par le feuillage provoque un blocage de la transpiration; l'énergie incidente est alors utilisée pour évaporer l'cau interceptée. 


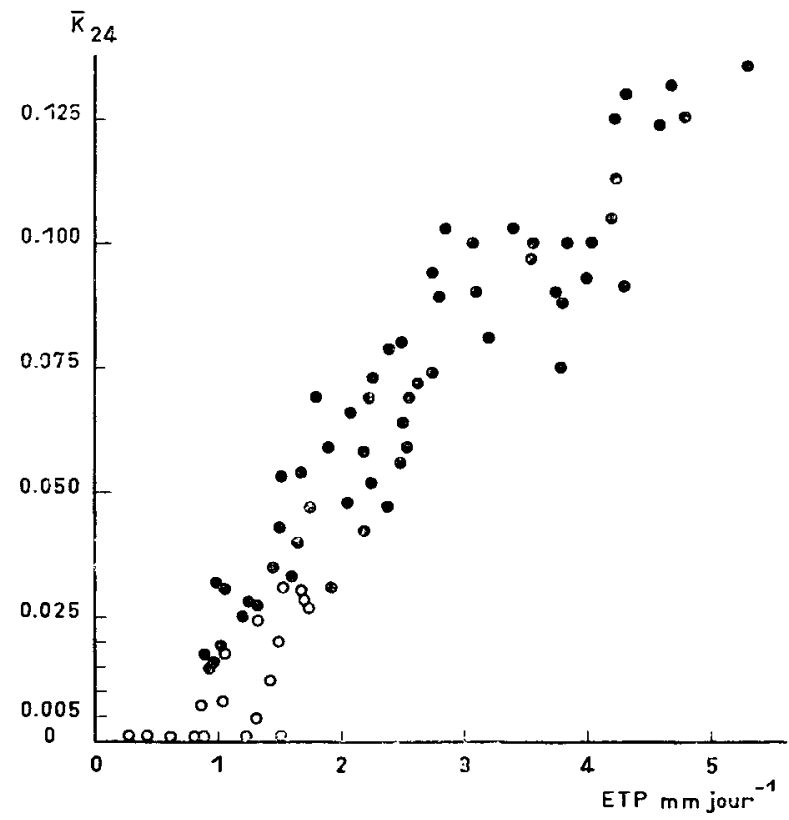

FIG. 4

Relation entre le coefficient $K_{24}$ (voir texte $\$ 2.2$ ) et l'E.T.P. Penman pour un Douglas en peuplement fermé.

Relationship between $K_{2_{i}}$ (see text $\$ 2.2$ ) and Penman P.E.T. for one Douglas-fir in a closed stand.

- Journées sans pluie.

Days without rain.

- Journées avec pluie.

Rainy days.

\section{Conclusion}

La méthode que nous avons décrite présente un certain nombre d'avantages. qui devraient permettre d'obtenir des résultats fiables, à deux niveaux :

- au niveau du fonctionnement hydrique de l'arbre, par létude fine des variations journalières du flux de sève, particulièrement en période de stress hydrique:

- au niveau du fonctionnement hydrique des peuplements foresticrs, sachant que la simplicité de mise en œuvre et le faible coût (2) de cette technique permettent d'aborder la mesure quantitative de la transpiration et de sa variabilité spatiale en forêt.

(2) Un capteur (dcux sondes) revient à environ $30 \mathrm{FF}$, le système d'amplification à $80 \mathrm{FF}$ par capteur. 
Lutilisation de cette méthode suppose, lorsqu'on désire calculer le flux total, de connaître la section du bois d'aubier au niveau du point de mesure. Selon le type d'essence et la précision souhaitée, on pourra estimer la section du bois d'aubier grâce à un ou plusieurs sondages à la tarière de Pressler ou la mesurer directement après abattage de larbre.

\section{Summary \\ A new method of sap flow measurement in tree stems}

The method described in this paper is based on a thermal sensor composed of two probes radially inserted in the sapwood of the trunk. One of those probes is heated at a constant energy and the other considered as at temperature reference. A simple equation enables us to calculate the sapflow as a function of the difference of the temperature between the two elements. A calibration has been made on pieces of trunk of different species.

Owing to its sensitivity and its low cost, this system may fit for the quantitative measurement of forests transpiration.

\section{Références bibliographiques}

Granier A., Gross P., 1984. Mesure du flux de sève brute chez le Douglas (Pseudotsuga menziesii Mirb. Franco) par la méthode thermo-électrique automatisée (à paraître).

HübER B.,SCHMidT E., 1937. Eine Kompensationsmethode zur thermoelecktrischen Messung langsamer Stafströme. Bcr. disch. Bot. Ges., 55, 514-529.

SWANSON R.H., 1962. An instrument for detecting sap movement in woody plants. Sta. Pap. Rocky Mt. For. Range Exp. Sta. n" 68.

SWaisson R.H., 1974. Velocitiy distribution patterns in ascending xylem sap during transpiration. In : Flow its Measurement and control in Science and Industry. Et. Rodger and Dowdell. Instrument Society of America. Vol. 1, 1425-30. 